\title{
Tuberculosis Status and Coinfection of Pulmonary Fungal Infections in Patients Referred to Reference Laboratory of Health Centers Ghaemshahr City during 2007-2017
}

\author{
Mohammad Reza Jabbari Amiri ${ }^{1}$, Rora Siami ${ }^{2}$, Azad Khaledi ${ }^{3,4 *}$
}

\author{
OPEN ACCESS \\ Citation: Mohammad Reza Jabbari \\ Amiri, Rora Siami, Azad Khaledi. \\ Tuberculosis Status and Coinfection of \\ Pulmonary Fungal Infections in Patients \\ Referred to Reference Laboratory of \\ Health Centers Ghaemshahr City during \\ 2007-2017. Ethiop J Sci.2018;28(6):683. \\ doi:http://dx.doi.org/10.4314/ejhs.v28i6.2 \\ Received: March 15, 2018 \\ Accepted: June 11, 2018 \\ Published: November 1, 2018 \\ Copyright: (C) 2018 Mohammed RA, et \\ al. This is an open access article \\ distributed under the terms of the \\ Creative Commons Attribution License, \\ which permits unrestricted use, \\ distribution, and reproduction in any \\ medium, provided the original author and \\ source are credited. \\ Funding: Nil \\ Competing Interests: The authors \\ declare that this manuscript was \\ approved by all authors in its form and \\ that no competing interest exists. \\ Affiliation and Correspondence: \\ ${ }^{1}$ Department of Medical Mycology and \\ Parasitology, Faculty of Medicine, \\ Mazandaran University of Medical \\ Sciences, Sari, Iran \\ ${ }^{2}$ Health Center, Ghaemshahr, Iran \\ ${ }^{3}$ Infectious Diseases, Research Center, \\ Kashan University of Medical \\ Sciences, Kashan, I.R. Iran \\ ${ }^{4}$ Department of Microbiology and \\ Immunology, School of Medicine, \\ Kashan University of Medical \\ Sciences, Kashan, I.R. Iran \\ *Email: azadkh99@gmail.com
}

\begin{abstract}
BACKGROUND: Pulmonary tuberculosis is one of the most important health concerns. Pulmonary fungal infections have clinical and radiological characteristics similar to tuberculosis which may be easily misdiagnosed as tuberculosis. This study aimed to evaluate tuberculosis (TB) status and coinfection of TB with pulmonary fungal infections in patients referred to reference laboratory of health centers Ghaemshahr city during 2007-2017.

METHODS: This cross-sectional study was conducted during eleven years, between 2007-2017, on 3577 patients with suspected TB referred to health centers of Ghaemshahr City. For isolation, sputum smear preparation and Ziehl-Neelson staining in companying with microscopy direct observation and $\mathrm{KOH} 10 \%+$ white Calcofluor staining was used. The culture of fungi was performed on Sabouraud Dextrose agar, Czapek and chrome agar media. Then, data were analyzed using SPSS software (version 16) through Student's t-test, Fisher test, and Odds Ratio. $P$ values $<0.05$ were considered statistically significant.

RESULTS: Of 3577 patients, 10731 smears were prepared, 3.6\% $(n=130)$ of patients were identified as smear-positive pulmonary tuberculosis, $86.4 \%(n=3090)$ were smear negative and $10 \%$ $(n=357)$ drug-resistant TB. The mean age of patients was $48 \pm 1.8$ years. With increasing age, the prevalence of $T B$ has increased which was statistically significant $(P$ value $<0.05)$. Prevalence of tuberculosis in the age group over than 57 years in males and females had an ascending trend. Amongst the positive tuberculosis patients, 16/130 cases (12.3\%) had the coinfection of TB with fungi microorganisms.

CONCLUSION: Our findings showed the coinfection of fungi agents in patients with tuberculosis that should be considered.

KEYWORDS: Coinfection, Mycobacterium tuberculosis, Pulmonary Fungal Infection
\end{abstract}




\section{INTRODUCTION}

Pulmonary tuberculosis is a major cause of mortality attributed to the Mycobacterium tuberculosis complex globally, tuberculosis (TB) is placed amongst the top 10 causes of death worldwide (1). More than $95 \%$ of pulmonary tuberculosis cases have been reported from developing countries, particularly from Asia, Africa, the Middle East and Latin America which have limited diagnostic and therapeutic equipment(2,3). The annual TB deaths have reported about three million people that two and one million of those have belonged to the adults and children, respectively(4). Currently, the incidence rate of smear-positive pulmonary tuberculosis in our country (Iran) is the main index in the measurement of tuberculosis situation. Tuberculosis incidence rate is counted as dividing the number of new cases of disease within a year in one hundred thousand populations (5). However, its incidence is highly variable by region. For example, in the United States, the annual incidence is less than 5 cases per 100,000 people, while in sub-Saharan Africa and Asia reaches to hundreds of cases per 100,000 people. The occurrence of TB is global. In 2016, the leading number of new TB cases was related to Asia, with $45 \%$ of new cases, followed by Africa, with $25 \%$ of new cases. In $2016,87 \%$ of new TB cases were from 30 high TB burden countries. Seven countries (India, Indonesia, China, Philippines, Pakistan, Nigeria, and South Africa) were responsible for $64 \%$ of the new TB cases (6).

Many risk factors such as war, immigration, family history of close contact with TB patients, social status, age, poverty, male gender, HIV infection, smoking, asthma and homelessness had the significant role in the risk of developing tuberculosis (7).

Multi-drug resistant TB (MDR-TB) is described as tuberculosis with resistance to both rifampicin and isoniazid drugs $(8,9)$. XDR-TB is a form of TB which is resistant to at least four of the core anti-TB drugs. XDR-TB involves resistance to the two most dominant anti-TB drugs, isoniazid, and rifampicin, also known as multidrug-resistance (MDR-TB), in addition to resistance to any of the fluoroquinolones (such as levofloxacin or moxifloxacin) and to at least one of the three injectable second-line drugs (amikacin, capreomycin or kanamycin) (10). Data obtained from the latest anti-TB drug resistance surveillance show that $4.1 \%$ of new and $19 \%$ of previously treated TB cases in the world are expected to rifampicin or multidrug-resistant tuberculosis (MDR/RR-TB), based on estimations in 2016, about 600000 new cases of MDR/RRTB emerged worldwide which caused 240000 deaths in the same year, especially in Asia. Also, nearly $6.2 \%$ of extensively drug-resistant $\mathrm{TB}$ (XDR-TB) was described, as well as, one million children developed TB and 250000 children died of TB in 2016 (11).

Sistan and Baluchestan, Khorasan, Mazandaran, Gilan, West Azerbaijan, East Azerbaijan, Ardabil, Kurdistan, and Khuzestan provinces are the regions of Iran with the high burden of tuberculosis (12). Based on the reports of Iranian Centre for Disease Control and Prevention, the number of confirmed MDR-TB cases has increased from 8 in 2003 to 41 in 2015 (13).

Filamentous saprophyte fungi have a wide distribution in nature, and their spore is abundant in the air and the transmission of infection occurs always through inhalation (14). The extensive use of antibiotics and steroids has recently caused a widespread prevalence of fungal pulmonary infection. Some factors such as immunodeficiency, chronic diseases, malignancy are involved in worsening the diseases and these fungal pulmonary infections may be formed primary and secondary in TB infection (15). In many cases, missed fungal pulmonary infection due to lack of specific clinical manifestations causes a high rate of morbidity and mortality (16). Candida albicans is the most prevalent fungal organism with the ability to causing infections, including slight mucocutaneous infection to invasive forms with affecting the multiple organs, sever Candida infections occur in immunosuppressive patients (17). The prevalence 
of $15-32 \%$ was reported in different studies of pulmonary tuberculosis coinfection with Candida (18). Four types of fungi: Aspergillus niger, A. fumigatus, Histoplasma capsulatum and Cryptococcus neoformans are main causes of malignant infections in lungs in patients affected by pulmonary tuberculosis (19).

Aspergillus is an airborne/saprophyte agent that does not normally form disease, but in immune-compromised people and also in individuals with damaged lungs such as those who have had TB can cause the disease. Because the symptoms of chronic pulmonary aspergillosis are very similar to TB symptoms that physician often misdiagnoses it, unfortunately, the infection can grow steadily and undetectably for years, and its treatment is too late successfully. Therefore, timely diagnosis of fungal diseases is of great importance in treating patients (20). Due to the lack of comprehensive studies about the epidemiology of TB and coinfection pulmonary fungal infections in patients from the Mazandaran province, Iran, this study aimed to evaluate the tuberculosis status and coinfection of pulmonary fungal infections in patients with TB referred to reference laboratory of health centers in Ghaemshahr City during 2007-2017.

\section{METHODS}

Patients and samples: In this cross-sectional study, information was extracted from medical records of patients with TB infection. The medical records were filled and completed by the educated and trained personnel in the health center of Ghaemshahr City. Three consecutive sputum samples were collected in a sterile dry widenecked, leak-proof container from each of the patients with suspected pulmonary tuberculosis referred to the TB health centers of Ghaemshahr City during eleven years (between 2007-2017), and they were transferred to Sari Medical School for microbiology and mycology staining. The patients referred were from urban and rural regions, prisoners, literate and illiterate. The study population included all new cases, recurrent $\mathrm{TB}$ and MDR-TB patients.

In this study, recurrent tuberculosis (TB), defined as $\mathrm{TB}$ that recurs after a patient has been considered cured. Also, patients who were not treated by first and second lines of anti TB drugs and still have signs and symptoms of tuberculosis and their smear were not negative considered as MDR-TB. For determination of sample size, Krejcie-morgan-sample-size-table was used. Based on the age variable, patients were categorized in 8 age groups $(0-10,11-20,21-30$, 31-40, 41-50, 51-60, 61-70, and 71-80).

Specimen processing and bacteriological procedures: The laboratory used of direct smear microscopy accompanying with culture in the diagnosis of Tuberculosis. Sputum specimens were homogenized with equal volumes of $50 \mathrm{mM}$ $\mathrm{NaOH}$ containing $\quad 0.5 \% \quad \mathrm{~N}$-acetyl-1-cysteine (NALC) and decontaminated with $10 \%$ sodium hydroxide, and then incubated at $37{ }^{\circ} \mathrm{C}$. Also, some of the specimens were used for ZiehlNeelson staining (21). And also, direct examination for identification of filamentous fungi was applied using staining white Calcoflura technique $+10 \% \quad \mathrm{KOH}$ through fluorescence microscopy with fungi culture in Sabouraud Dextrose agar medium containing chloramphenicol (SC) with incubating at $30^{\circ} \mathrm{C}$ for a week. Aspergillus species were identified using sub-culture on Czapek agar medium with diagnosis keys provided by Raper and Fennell previously. Diagnosis of yeasts was conducted by sub-culture on Chrom agar/Corn meal agar with Tween $80 \%$ and the germ tube test. Clinical and radiographic parameters were evaluated in all patients with tuberculosis. The co-infection cases of fungi with TB were reported according to 8 different age groups.

The quality control of the laboratory in the diagnosis of tuberculosis was carried out by annual quality control samples. As well as for further quality, the positive and negative sputum samples were transferred to the capital of Mazandaran Province (Sari).

Ethical considerations: Because the study had a laboratory base, did not require the specific ethical considerations. However, all patients' rights were respected, such as the non-disclosure of their names.

Statistical analysis: Data were analyzed using SPSS software (version 16) by Fisher's exact

DOI: http://dx.doi.org/10.4314/ejhs.v28i6.2 
test, and Odds Ratio. $P$ values of less than 0.05 were regarded as statistically significant.

\section{RESULTS}

From a total of 3577 patients with suspected TB during ten-years, 10731 smears were prepared. Of which $30(0.28 \%), 53(0.49 \%)$ and $19(0.18 \%)$ had respectively, one, two and three positive sputum smears. Also, 3.6\% $(\mathrm{n}=130)$ of patients were identified as smear-positive pulmonary tuberculosis. And, 86.4\% ( $\mathrm{n}=3090)$ were smearnegative pulmonary tuberculosis and 10\% $(n=357)$ were drug-resistant TB. Based on the findings of this study, the prevalence of tuberculosis in males was more than in females. And, the percentages of male and female with smear-positive tuberculosis were reported as $70 \%$ (91/130) and 30\% (39/130), respectively. And, a significant statistical correlation was seen between gender and infection with TB (P-value $<0.05)$. With increasing age, the prevalence of TB has increased, and this increase was statistically significant $(\mathrm{P}$-value $<0.05)$. The mean age of patients was $48 \pm 1.8$ years and the minimum and maximum ages of patients were 10 and 86 years, respectively. The highest incidence was found in patients aged 36-68 years. It should be noted that the prevalence of tuberculosis in the age group over 57 years in both sexes had an ascending trend. Totally, the trend of tuberculosis from 2007 to 2017 has a descending trend. The most symptoms of pulmonary TB in patients included in this study were cough, consistent fever, low- grade fevers, night sweats, loss of appetite, and chest pain.

In total, 48/10731 smears $(0.44 \%)$ were positive for fungi infections. Amongst the positive tuberculosis patients, $16 / 130$ cases $(12.3 \%)$ had the coinfection of Mycobacterium tuberculosis with fungi microorganisms such as Candida albicans, Aspergillus fumigatus and, A. flavus. Ten out of 16 cases $(62.5 \%)$ had the coinfection Aspergillus spp., with TB and $6 / 12(37.5 \%)$ had the coinfection of Candida spp., with TB. As shown in Figure 1, the most prevalence of coinfection of fungi with TB $(31.2 \%, 5 / 16)$ was in the age group of 41-50 years. With increasing age, the prevalence of coinfection TB with fungi infection has increased, and this increase was statistically significant ( $\mathrm{P}$ value $<0.05$ ), because $56.2 \%(9 / 16)$ cases of coinfection were in patients greater than 40 years. The most frequency of coinfection of Aspergillus spp., with TB (18.7\%, $3 / 16)$ was observed in age groups (41-50) and (51-60). And also, the most occurrence of coinfection of Candida spp., with TB (12.5\%, 2/16) was reported in the age groups of (21-30) and (41-50). In this study, amongst Aspergillus spp., A. fumigatus $(43.75 \%)$ was the most frequent, and non-albicans spp. (25\%) were the most common species amongst Candida spp. recovered from sputum samples. The coinfection of TB with fungal infections in males $(15.4 \%)$ was higher than in females $(14.7 \%),(\mathrm{P}=0.01)$. Overall, tuberculosis could be a risk factor for fungal infection (Odds ratio $=.32 ; 95 \%$ CI for $\mathrm{OR}=.10$ to 1.00 ). The further details related to the prevalence of fungi spp, are listed in the Table1.

Table1: Prevalence of fungi spp. in male and females based on the age groups.

\begin{tabular}{|c|c|c|c|c|c|c|}
\hline \multirow[t]{2}{*}{$\begin{array}{l}\text { No. of patients with } \\
\text { coinfection of fungi with TB }\end{array}$} & \multicolumn{3}{|c|}{ Aspergillus spp. $($ Total $=10)$} & \multicolumn{2}{|c|}{ C. albicans spp. $($ Total $=6)$} & \multirow{2}{*}{$\begin{array}{l}\text { T-test } \\
P \text { - } \\
\text { value }\end{array}$} \\
\hline & $\begin{array}{l}\text { A. } \\
\text { fumigatus }\end{array}$ & A. flavus & A. niger & $\begin{array}{l}\text { C. } \\
\text { albicans }\end{array}$ & $\begin{array}{l}\text { None } C . \\
\text { albicans spp. }\end{array}$ & \\
\hline $\operatorname{Male}(\mathrm{n}=91)$ & $5(31.25 \%)$ & $1(6.25 \%)$ & $1(6.25 \%)$ & $1(6.25 \%)$ & $3(18.75 \%)$ & 0.01 \\
\hline $\operatorname{Female}(n=49)$ & $2(12.5 \%)$ & $1(6.25 \%)$ & $0(0 \%)$ & $1(6.25 \%)$ & $1(6.25 \%)$ & 0.61 \\
\hline Total & $7(43.75 \%)$ & $2(12.5 \%)$ & $1(6.25 \%)$ & $2(12.5 \%)$ & $4(25 \%)$ & - \\
\hline
\end{tabular}

DOI: http://dx.doi.org/10.4314/ejhs.v28i6.2 


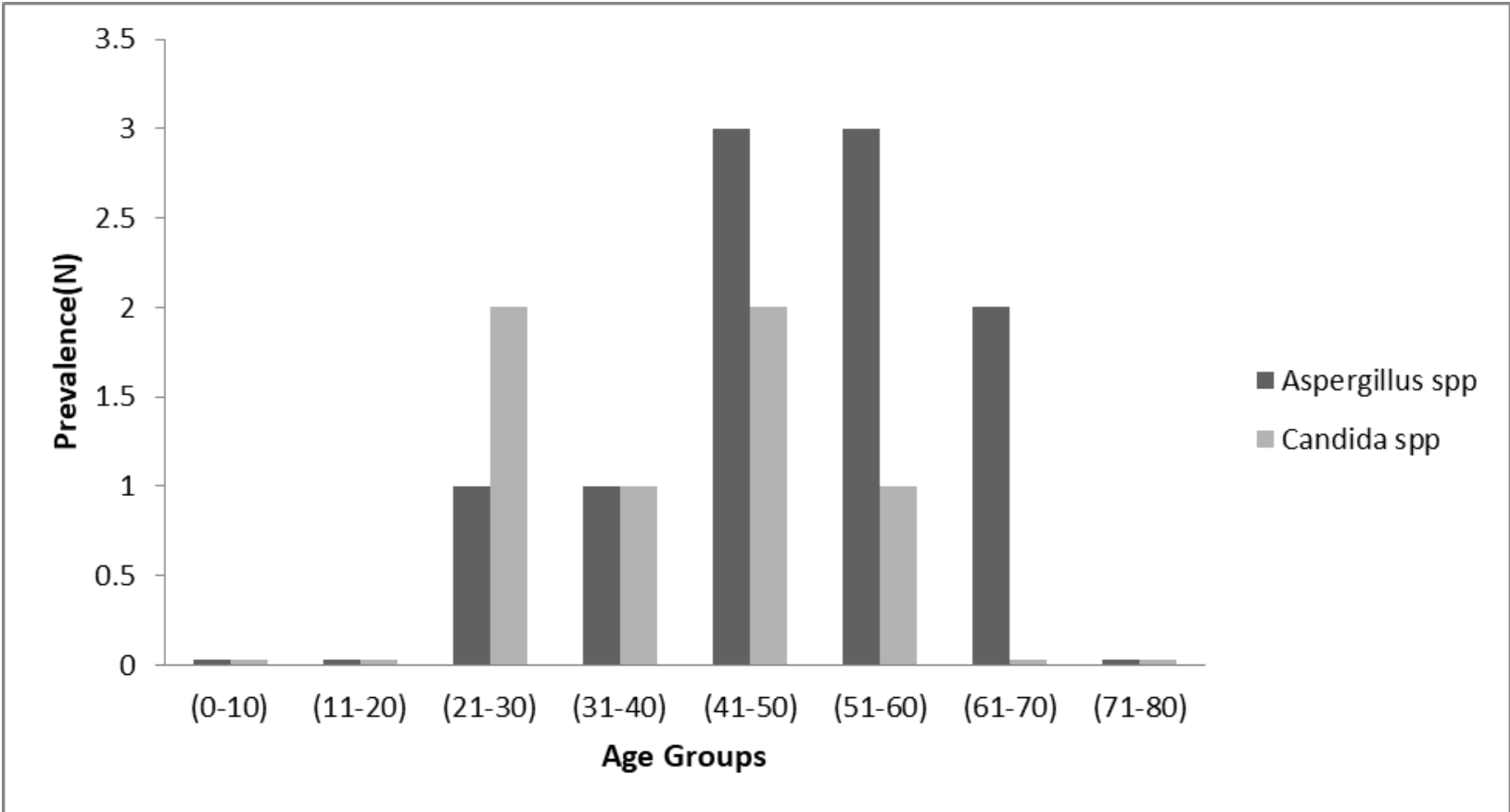

Fig. 1. Prevalence of fungi spp. in male and female based on the age groups.

\section{DISCUSSION}

Tuberculosis is a serious health and treatment problem which occurs in all countries over the world, but in terms of condition, its prevalence is very different, the distribution of incidence and prevalence rate of TB is not the same in all areas of our country (Iran). A study conducted by Manzouri, et al. showed that, between 2005 and 2006 years, the percentage of new smear-positive pulmonary TB prevalence was $11.1 \%$ (22). In a study conducted by Gholami, et al., during 20022007 years, in Uremia (Iran) showed that among 248 positive patients for tuberculosis, 151 and 97 cases were respectively smear-positive and smear negative. Also, $66.1 \%$ of those were male. In line to their findings, our results revealed that the 130 cases were smear positive and $46.5 \%$ of patients were male (23). In contrast to our results, in a study carried out by Mousavi, et al., during 20012007 years in Kashan, among the 196 TB patients, $67 \%$ of patients were pulmonary tuberculosis, and most of the patients with pulmonary tuberculosis were female (24). In addition, in a study conducted by Ebrahim Zadeh, et al., during 1995-
2006 years showed that among 840 patients in Birjand, $46.2 \%$ were male and $70 \%$ cases were pulmonary tuberculosis (25).

In a study conducted by Rafiee, et al., in Golestan Province(Iran), during 2005-2006, on patients with extra and pulmonary $\mathrm{TB}$, their findings showed that the number of the males with smear-positive pulmonary tuberculosis was higher than females. And also the incidence of TB was increased with age (26). Similar to our results, the ratio of males/females was higher. A significant correlation was found between the incidence of TB with increasing the age. But in contrary to our results, the rate of pulmonary tuberculosis cases was very higher. This difference can probably be attributed to the fact that the samples in their studies were collected from all parts of the Golestan province, but our samples were collected only from Ghaemshahr City (located in Mazandaran province), and they counted both pulmonary and extrapulmonary TB together, while we considered only pulmonary $\mathrm{TB}$, of course. It should be noted that the province of Golestan alongside Sistan and Baluchestan are two provinces with the high burden of $\mathrm{TB}$ than

DOI: http://dx.doi.org/10.4314/ejhs.v28i6.2 
other regions of Iran (27). In agreement to several studies conducted in different parts of our country (Iran), the prevalence of TB in males was higher than in females, which can be attributed to the more attendance of male in the community and more close contacts with carriers/patients and the risk factors that lead to tuberculosis (28). Also, in this study, the mean age of patients was $48 \pm 1.8$ years. The highest incidence was found in patients aged 36-68 years. The prevalence of tuberculosis in the age group of over 57 years in both sexes had an ascending trend. These findings are consistent with reports from other studies. This ascending trend of tuberculosis prevalence in aged people is due to reactivation of latent TB in these patients (29).

Totally, the trend of tuberculosis from 2007 to 2017 has a descending trend. However, in contrast to our results, a study conducted in Singapore, did not show a decreasing trend in the number of TB patients from 2006 to 2010 . Similarly, from 2011 to 2013, this increased number of TB cases probably resulted from Medical tourism and the high flood of foreign workers to Singapore (30).

The prevalence of opportunistic fungal infections has recently increased, which normally incapable of causing disease in healthy persons. While they are the potential pathogens in immunocompromised individuals, patients with underlying diseases, broad consumption of antibiotics, impairments of immunity in pulmonary tuberculosis patients (31). In this study, as mentioned in the results section, $A$. fumigatus, $A$. flavus and $A$. niger plus nonalbicans spp., were the most common species recovered from sputum samples. In line with our findings, in the study conducted by N.M. Osman and et al., in Egypt, in 2012 on 50 tuberculosis patients, A. fumigatus was reported as the most frequent Aspergillus spp., followed by A. niger and $A$. flavus, while other fungal species were not detected (15). Also, other studies from over the world reported the Aspergillus spp., as the predominant fungi in patients suspected of pulmonary tuberculosis (32-34). The leading Aspergillus spp., detected from sputum in this study was A. fumigates, while Razmpa et al., reported the higher prevalence for A. flavus (35). In the study conducted at Patna Medical College Hospital, Bihar (India), in 2011 to 2012, 75 sputum samples were collected from patients suspected of tuberculosis. Their results indicated that the Candida albicans was isolated in $44.4 \%$ of cases followed by $A$. niger with a prevalence of $33.3 \%$ (36), as well as others reported the $C$. albicans as the most frequent fungi spp (37). The correlation between co-existence of Candida infections and pulmonary tuberculosis has increased concern during recent years (38). In contrary to their findings, our results showed the Aspergillus spp., as the most predominant fungi and also, amongst Aspergillus spp., A. fumigatus was predominant. The findings of a study carried out by Speirs et al., showed that the A. fumigatus was the common agent of chronic respiratory infections such as M. tuberculosis. This is consistent with our results. In addition, a variety of studies have shown that pulmonary colonization of potentially pathogenic microorganisms such as Aspergillus in some patients with COPD, cystic fibrosis or tuberculosis is associated with high mortality and inappropriate prognosis (39). The results of this study indicated that the situation of tuberculosis disease could be one of the risk factors for fungal infections. Several studies also have shown that $A$. fumigatus like $M$. tuberculosis has colonized respiratory tract of patients with respiratory complications (40).

As presented in our study, the coinfection of TB with fungal infections in males was higher than in females. This is possibly attributed to the fact that the males are more susceptible to infection than females owing to their higher exposure to the surrounding as stated in a study conducted by Bansod et al., in which fungal infection was reported higher in males than in females (19).

Regarding the role of fungal agents in exacerbation of tuberculosis and the lack of improvement in tuberculosis symptoms such as chronic cough, sputum or occasional hemoptysis following completion of anti-tuberculosis 
chemotherapy, and miss diagnosis with tuberculosis, it is necessary to pay more attention to this topic. It would be used of more accurate molecular techniques in the diagnosis of fungal agents especially in coinfection of those with pulmonary tuberculosis.

\section{ACKNOWLEDGMENT}

We would like to thank our collogues from Laboratory of health centers Ghaemshahr City for helping in collecting the data.

\section{REFERENCES}

1. Society AT. Diagnostic standards and classification of tuberculosis in adults and children. Am $J$ Respir Crit Care Med. 2000;161:1376-95.

2. Organization WH. Global tuberculosis report 2013: World Health Organization; 2013.

3. Amiri MJ, Karami P, Chichaklu AH, Jangan EH, Amiri MJ, Owrang M, et al. Identification and Isolation of Mycobacterium tuberculosis from Iranian Patients with Recurrent TB using Different Staining Methods. J Res Med Dent Sci. 2018;6(2):409-14.

4. Tiwari R, Tiwari D, Garg SK, Chandra R, Bisen PS. Glycolipids of Mycobacterium tuberculosis strain $\mathrm{H} 37 \mathrm{Rv}$ are potential serological markers for diagnosis of active tuberculosis. Clin Diagn Lab Immunol. 2005;12(3):465-73.

5. Nasehi M, Mirhaghani L. National tuberculosis control guide. 2nd Tehran: Andishmand Publisher. 2009:5-21.

6. Organization WH. Global Tuberc Repor. 2016. 2016.

7. Babamahmoodi F, Alikhani A, Yazdani Charati J, Ghovvati A, Ahangarkani F, Delavarian L, et al. Clinical epidemiology and paraclinical findings in tuberculosis patients in north of Iran. BioMed Res Int. 2015;2015.

8. Tadolini M, Lingtsang RD, Tiberi S, Enwerem M, D'Ambrosio L, Sadutshang TD, et al. First case of extensively drug-resistant tuberculosis treated with both delamanid and bedaquiline. Europe Resp J. 2016;48(3):935.

9. Ashna H, Kaffash A, Khaledi A, Ghazvini K. Mutations of rpob Gene Associated with Rifampin Resistance among Mycobacterium Tuberculosis Isolated in Tuberculosis Regional
Reference Laboratory in Northeast of Iran during 2015-2016. Ethiop $J$ Health Sci. 2018;28(3):299-304.

10. Organization WH. WHO treatment guidelines for drug-resistant tuberculosis 2016 update: World Health Organization; 2016.

11. Organization WH. Guidelines for the treatment of drug-susceptible tuberculosis and patient care, 2017 update. 2017.

12. Bijari B, Sharifzade GR, Abbasi A, Salehi S. Epidemiological survey of animal bites in east of Iran. Arch Clin Infect Dis. 2011;6(2):90-2.

13. Heidary M, Nasiri MJ. Why has MDR-TB prevalence increased in Iran? J Clin Tuberc Other Mycobact Dis. 2016;5:9.

14. Hedayati MT, Mayahi S, Denning DW. A study on Aspergillus species in houses of asthmatic patients from Sari City, Iran and a brief review of the health effects of exposure to indoor Aspergillus. Environ Monit Assess. 2010;168(14):481-7.

15. Osman NM, Gomaa AA, Sayed NM. Microarray detection of fungal infection in pulmonary tuberculosis. Egypt $J$ Chest Dis. 2013;62(1):151-7.

16. Pfaller M, Diekema D. Epidemiology of invasive candidiasis: a persistent public health problem. Clin Microbiol Rev. 2007;20(1):13363.

17. Chen X-H, Gao y-C, Zhang Y, Tang z-H, YU Y-S, Zang G-Q. Tuberculosis infection might increase the risk of invasive candidiasis in an immunocompetent patient. Rev Inst Med Trop Sao Paulo. 2015;57(3):273-5.

18. Fontalvo DM, Jiménez Borré G, Gómez Camargo D, Chalavé Jiménez N, Bellido Rodríguez J, Cuadrado Cano $\mathrm{B}$, et al. Tuberculosis and fungal co-infection present in a previously healthy patient. Colombia Médica. 2016;47(2):105-8.

19. Bansod S, Rai M. Emerging of mycotic infection in patients infected with Mycobacterium tuberculosis. World J Med Sci. 2008;3(2):74-8.

20. Guinea J, Torres-Narbona M, Gijón P, et al. Pulmonary aspergillosis in patients with chronic obstructive pulmonary disease: incidence, risk factors, and outcome. Clin Microbiol Infect. 2010;16(7):870-7.

DOI: http://dx.doi.org/10.4314/ejhs.v28i6.2 
21. Nakhjavani F, Bahador A. Direct detection of Mycobacterium sp in respiratory specimen with rpoB-PCR and comparison with concentration fluorochrome staining. Res J Med Med Sci. 2006;1(2):68-71.

22. Manzouri L, Farajzadegan Z, Babak A, Farid F, Fadaeinobari R. Tuberculosis Program Evaluation in Isfahan District. $J$ Isfahan Med Sch. 2010;27(102).

23. Gholami A, Gharah AR, Mousavi JL, Sadaghianifar A. Epidemiologic survey of pulmonary tuberculosis in Urmia city during 2004-2007. 2009.

24. Ghorbani F, Shadkam M, Vojdani S, Pakide M. A comparative study of patients with pulmonary tuberculosis and extra-pulmonary tuberculosis in Kashan. KAUMS Journal (FEYZ). 2009;13(3):235-41.

25. Ebrahimzadeh A, Sharifzadeh GR, Eshaghi S. The epidemiology of Tuberculosis in Birjand (1996-2006). $J$ Birjand Un Med Sci. 2009;16(1):31-8.

26. Rafiei S, Besharat S, Jabari A, Golalipour F, Naser MA. Epidemiology of tuberculosis in northeast of Iran: a population-based study. 2009.

27. Rokni F, Etemadi J. An epidemiological study of extrapulmomary tuberculosis in Mashhad (1997-2001). 2003.

28. Ayatollahi J, Sharifi M, Razmi H, Mahmoodiardekani B. The survey of tuberculosis patients in Yazd and Shiraz. 2010.

29. Ai J-W, Ruan Q-L, Liu Q-H, Zhang W-H. Updates on the risk factors for latent tuberculosis reactivation and their managements. Emerg Microbes Infect. 2016;5(2):e10.

30. Jappar SB, Low SY. Tuberculosis trends over a five-year period at a tertiary care universityaffiliated hospital in Singapore. Singapore Med J. 2015;56(9):502.

31. Köhler JR, Casadevall A, Perfect J. The spectrum of fungi that infects humans. Cold Spring Harb Perspect Med. 2015;5(1):a019273.

32. Njunda AL, Ewang AA, Kamga L-HF, Nsagha DS, Assob J-CN, Ndah DA, et al. Respiratory tract Aspergillosis in the sputum of patients suspected of tuberculosis in Fako divisionCameroon. J Microbiol Res. 2012;2(4):68-72.
33. Kurhade A, Deshmukh J, Fule R, Chande C, Akulwar S. Mycological and serological study of pulmonary aspergillosis in central India. Indian J Med Microbiol. 2002;20(3):141.

34. Ekenna O, Uba A, Chikwem J, Mambula S, Aliyu M, Mohammed I. Relevance of moldy fungi as agents of chronic lower respiratory tract infection in patients seen in Maiduguri, Nigeria. West Afr J Med. 2007;26(2):117-20.

35. Razmpa E, Khajavi M, HADIPOUR JM, Kordbacheh P. The prevalence of fungal infections in nasal polyposis. 2007.

36. Babita SS, Prabhat K. Prevalence of mycotic flora with pulmonary tuberculosis patient in a tertiary care hospital. Int J Contemporary Med Res. 2016;3(9):2563-4.

37. Kali A, Charles MP, Noyal MJ, Sivaraman U, Kumar S, Easow JM. Prevalence of Candida coinfection in patients with pulmonary tuberculosis. Australas Med J. 2013;6(8):387.

38. Naz SA, Tariq P. A study of the trend in prevalence of opportunistic Candidal coinfections among patients of pulmonary tuberculosis. Pak J Bot. 2004;36:857-62.

39. Speirs JJ, van der Ent CK, Beekman JM. Effects of Aspergillus fumigatus colonization on lung function in cystic fibrosis. Curr Opin Pulm Med. 2012;18(6):632-8.

40. He H, Chang S, Ding L, Sun B, Li F, Zhan Q. Significance of Aspergillus spp. isolation from lower respiratory tract samples for the diagnosis and prognosis of invasive pulmonary aspergillosis in chronic obstructive pulmonary disease. Chin Med J. 2012;125(17):2973-8. 